\title{
1 Geschichte und Geschichten
}

Tief im Schoße des Kyffhäusers

Bei der Ampel rotem Schein

Sitzt der alte Kaiser Friedrich

An dem Tisch von Marmorstein. ${ }^{1}$

Mit diesen Worten beginnt Franz Emanuel August von Geibels Gedicht Friedrich Rotbart. Er zitiert damit das Bild, das sich die Nachwelt - hier speziell die des 19. Jahrhunderts - von Kaiser Friedrich I. Barbarossa gemacht hat; daneben existiert ein historiographisches, ,wissenschaftliches ${ }^{6}$ - und ebenso veränderbares - Bild des Kaisers und seiner Taten. ${ }^{2}$ Die Erinnerung an den historischen Kaiser bleibt offensichtlich nicht dabei stehen, die Ereignisse aus seinem Leben zu bewahren, sondern sie bringt ihn schließlich als einen Unsterblichen in die sagenhafte Welt des Kyffhäusers, in dem er sitzt und wartet, bis der Zeitpunkt seiner Wiederkehr gekommen ist. So mündet historiographisches Erinnern an Geschichte in literarische Überformung von Geschichte. Es ist unzweifelhaft, dass der im Kyffhäuser wartende ,Kaiser Rotbart' mit einem historisch nachweisbaren Herrscher des 12. Jahrhunderts in der Stofftradition zu identifizieren ist, auch wenn ebenso deutlich ist, dass es sich bei der Wiederkehr des mittelalterlichen Kaisers um eine Sage handelt. Eine Vermischung von Historischem und ,Sagenhaftem ' findet sich etwa auch bei der Figur des Tannhäuser, dessen, Geschichte‘ über die Rezeption Richard Wagners bis ins 21. Jahrhundert gelangt ist. Der historische Lieddichter und Minnesänger Tannhäuser aber ist nur noch Fachleuten bekannt und er hat darüber hinaus mit der Figur aus Wagners Oper noch weniger zu tun als Friedrich I. Barbarossa mit ,Kaiser Rotbart ${ }^{6}{ }^{3}$ Auch Wagners Figur ist ein Sänger (aus einem wie auch immer gearteten ,Mittelalter'), doch wie und warum gerade dieser Sänger (in dieser Form greifbar durch Richard Wagner) ${ }^{4}$ mit dem Wartburgkrieg 5 und mit der Venusberg-Sage verbunden wird, ${ }^{6}$ wird wohl nie ganz zu klären sein. Ähnliches trifft auf den ,edlen Moringer' der Moringer-Ballade zu, dessen Geschichte heute außerhalb der mediävistischen Fachwelt wohl ebenso unbekannt ist wie der historische Minnesänger Heinrich von Morungen selbst. ${ }^{7}$ Mit Theoderich dem Großen ist ein weiterer Herrscher des (Früh-)Mittelalters greifbar, dessen ,Weg in die Geschichte' noch weiter geht als der Barbarossas: Als Dietrich von Bern gelangt er nicht nur als Protagonist in eine Erzähltradition, die an tatsächliche Ereignisse um Theoderich den Großen anknüpft - die sogenannte bistorische Dietrichepik -, sondern wird darüber

1 Geibel (1920), S. 84

2 Vgl. dazu Johannes Fried: „Friedrich Barbarossa, der ,Kaiser Rotbart lobesam“ (Ludwig Uhland), galt lange Zeit als deutscher Held, als Friedensstifter, Einheitsbringer und Erretter aus größter Not. Doch allmählich bröckelt sein Ruhm, wird deutlich, daß er ein verschlagener, skrupelloser Machtpolitiker war ...“ (J. Fried [2013], S. 153).

3 Vgl. zur Rezeption Barbarossas im 19. und 20. Jahrhundert Kaul (2007), zur Rezeption Tannhäusers in der Tannhäuser-Sage Rüther (2007).

4 Die Quellenlage ist hier nicht eindeutig: Wagner selbst benennt eine Quelle für diese (zunächst wohl lose) Zusammenführung, die aber ,von der Forschung bisher nicht nach[zu]weisen“ (V. Mertens [1986], S. 22) ist; nach Mertens liegt es allerdings (sehr) nahe, dass Wagner einen Text Ludwig Bechsteins verwendet habe, der ebenfalls beide Komplexe in Verbindung bringt (vgl. V. Mertens [1986], S. 22).

5 Im Wartburgkrieg, einem „Komplex von mehreren Dichtungen des 13. bis 15. Jh.s“ (Wachinger [1999], Sp. 740), treten neben dem (wohl) fiktiven Heinrich von Ofterdingen die (literar-)historisch (mehr oder weniger gut) bezeugten Lieddichter Walther von der Vogelweide, Wolfram von Eschenbach, Reinmar von Zweter, Biterolf und der Tugendhafte Schreiber auf, nicht aber der Tannhäuser.

6 Als eines der ältesten Zeugnisse dieser Verbindung des Tannhäusers mit der Venusberg-Sage gilt die TannhäuserBallade aus dem 15. Jahrhundert (vgl. Wachinger [1995], Sp. 614).

7 Vgl. auch dazu Rüther (2007). 
hinaus im heldenepischen Kosmos des Mittelalters eine zentrale, stark typisierte Figur, die sich - in der sogenannten âventiurehaften Dietrichepik - zu verselbständigtem seriellen Erzählen weitab von jeder historischen Basis eignet und vor allem auch auf diesem Weg bis in die Neuzeit hinein bekannt bleibt. ${ }^{8}$ Eine ähnliche ,Karriere' war dem aus dem Nibelungenlied bekannten Siegfried beschieden (dessen historische Wurzeln freilich höchst vage sind), indem er - v. a. auch in seiner Rolle als Drachentöter - als eine der wenigen mittelalterlichen Figuren auch über die Zeit des Buchdrucks hinaus bis ins 18. Jahrhundert als ,gehörnter 'Siegfried präsent blieb. ${ }^{9}$

Allen diesen Geschichten gemeinsam ist die Überlagerung von Historie und Sage, z. T. auch die Vermischung von historischen Daten und Fakten verschiedener Personen, wobei die Verortung der Geschichten auf der Skala zwischen (historischem) ,Fakt` und (literarischer), Fiktion sehr unterschiedlich ausfallen kann; gleiches gilt für die Frage, inwieweit die literarische Überformung neben ein historisch fundiertes Bild tritt (Friedrich I. Barbarossa) oder dieses sogar gänzlich überlagert (Tannhäuser). Das Interesse an einer solchen Form des (kollektiven) Erinnerns an historische Ereignisse und/oder Personen ist zwar sicherlich nicht erst eine Erscheinung des Spätmittelalters bzw. der Frühen Neuzeit, doch die Tendenzen zu ,Geschichten über Geschichte‘ bzw. das Interesse an Erzählungen in dem genannten Spannungsfeld zwischen ,Fakt ${ }^{`}$ und ,Fiktion scheinen im 15. und 16. Jahrhundert sehr ausgeprägt gewesen zu sein und zwar sowohl im engeren familiären (dynastischen) als auch im übergreifenden politischen (im weitesten Sinne ,nationalen) Bereich..$^{10}$ Die genannten Beispiele - Tannbäuser- und Moringer-Ballade, Wartburgkrieg, aventiurebafte Dietrichepik, der gehörnte Siegfried - entwickeln sich ab dem 13. bis ins 15. Jahrhundert und werden teilweise noch lange weitertradiert. Dies zeigt beispielhaft etwa auch die Geschichte von Herzog Emst $t^{11}$, deren älteste literarisierte Überlieferung aus dem 12. Jahrhundert stammt; es ist aber vermutlich kein Zufall, dass gerade diese Geschichte auch im Zeitalter des Buchdrucks sehr populär ist. ${ }^{12}$ Auch für die Geschichtsschreibung lassen sich für das Spätmittelalter und die Frühe Neuzeit an etlichen Beispielen „das Streben nach Kurzweil und ein Hang zum Fabulieren“13 und damit „eine Auflockerung im Verhältnis zur historischen Wirklichkeit"“14 feststellen; demgegenüber besteht offenbar ein Bedürfnis nach Historisierung von ,Geschichten', das durch die Anbindung von (fiktiven) Erzählungen an historische Personen, Orte oder Dynastien bedient wird. ${ }^{15}$

Während heldenepische Texte, die in der Regel im 12. und 13. Jahrhundert verschriftlicht wurden, Personen und Ereignisse aus der Völkerwanderungszeit verarbeiten, wobei der jahrhun-

8 Dietrich von Bern war bis weit in das 20. Jahrhundert hinein über Sagensammlungen auch weiterhin präsent; mit dem Verschwinden bzw. dem Rückgang dieser Sagensammlungen schwindet auch die Bekanntheit Dietrichs von Bern. In der Frühen Neuzeit gelangen ,gerade die aventiurehaften, also diejenigen [Dietrichepen] ohne historischen Gehalt“ (Braun [2004], S. 345), in den Druck (in Prosa-Versionen), nicht die Texte der historischen Dietrichepik.

9 Einen Überblick über die zentralen Werke im Umkreis von Nibelungen- und Dietrich-Epik bietet Brunner (2010), S. $252 \mathrm{f}$.

10 Ähnliches lässt sich wohl für die Gegenwart sagen - wenn auch mit einer Tendenz, die deutlich weiter in die Vergangenheit reicht -, wenn man auf die Popularität von Historischen Romanen zu mittelalterlichen Personen und Ereignissen blickt, wobei die Implikationen die Behauptung von, Wahrheit betreffend trotz eines ,Fiktionsvertrages sicherlich andere sind (magische oder Anderwelt-Elemente etwa sind als nicht real denkbare Phänomene Teil von Fantasy-Geschichten, in der Regel aber nicht von Historischen Romanen). Zu Entstehung und Entwicklung ,unser[es] gegenwärtige[n] System[s] an Fiktions- oder Wahrheitsverträgen" mit Blick auf das 15. und 16. Jahrhundert vgl. Ziegeler (1999), Zitat S. 238.

11 Vgl. dazu Szklenar/Behr (1981).

12 Vgl. Die Historie von Herzog Ernst (1992).

13 Winter (1999), S. 43.

14 Winter (1999), S. 44

15 Vgl. dazu Hellmuth (1991), S. 140-144. 
dertelange Weg, den die Stoffe in der Mündlichkeit genommen haben, von uns nicht nachvollzogen werden kann, ist die für uns greifbare Spanne, innerhalb derer Personen wie der Tannhäuser mit Sagenstoffen in Verbindung gebracht und damit literarisch erinnert werden, deutlich kürzer. Eine gewisse zeitliche Distanz scheint aber in der Regel dennoch notwendig zu sein, um historische ,Fakten' in der literarischen (Neu-)Formulierung ignorieren zu können - auch wenn gleichwohl beide Traditionen, die historiographische und die literarische, nebeneinander existieren können. Das 16. Jahrhundert bietet mit den Möglichkeiten des Buchdrucks und dem (beginnenden) Humanismus ein Feld sowohl für die Entstehung und Verbreitung von ,Sagen`als auch für die ,wissenschaftliche ' historiographische Beschäftigung mit der Vergangenheit und dies umso mehr, als zum einen die Möglichkeit zur literarischen Produktion zwar noch immer Finanzkraft erfordert, dies aber in deutlich geringerem Maße als in Zeiten der Pergament-Handschriften-Produktion, ${ }^{16}$ und zum anderen das Verfassen von Texten nicht mehr zwangsläufig (wenn auch noch häufig) mit dem Auftrag durch einen Gönner verbunden ist. Ein Blick auf Texte dieser Zeit verspricht somit ein (noch) differenzierteres Bild in Hinblick auf den Weg von ,Geschichte' in ,Geschichten`.

\subsection{Die Relation von Historie und Literatur}

Erzählen - episches Erzählen - ist zunächst und über viele Jahrhunderte Erzählen von etwas Vergangenem. Erzählverfahren, die eine direkte Augen- oder Ohrenzeugenschaft suggerieren, also sozusagen zeitgleich zum Geschehen berichten, sind relativ jung; zu denken ist hier etwa an den Kunstgriff des inneren Monologs als eine Form des ,zeitdeckende[n] Erzäblen $[s]^{\text {"1 } 17}$, der in Arthur Schnitzlers Fräulein Else ${ }^{18}$ (erschienen 1924) die gesamte Erzählung bildet, oder an den Roman Das Wetter vor 15 Jahren (erschienen 2006) von Wolf Haas, der von der ersten bis zur letzten Zeile aus einem Interview eines Autors (Wolf Haas genannt) mit einem Reporter der ,Literaturbeilage besteht. ${ }^{19}$ Wie weit das zu Erzählende jedoch in der Vergangenheit zurückliegt, ist völlig variabel. In vielen Fällen, wie etwa auch im mittelhochdeutschen höfischen Roman, bleibt (absichtlich) im Unklaren, wann die erzählte Handlung stattgefunden haben soll. Im Iwein Hartmanns von Aue heißt es beispielsweise über König Artus:

sî [die lantliute; A. S.] jehent er lebe noch hiute:

er hât den lop erworben,

ist im der lip erstorben,

sô lebet doch iemer sîn name.

(I 14-17) ${ }^{20}$

Der Rezipient erfährt also, dass mit König Artus zumindest ein Protagonist der Erzählung nicht mehr am Leben ist; dieser wird in einer sagenhaften Zeit, einer Art Goldenem Zeitalter, verortet

6 Vgl. dazu J.-D. Müller (1982), S. 76.

17 Schönhaar (1990), S. 138 (Herv. i. O.).

18 Vgl. Schnitzler (2008).

19 Vgl. Haas (2007b). Am Ende seines vorherigen Buches, Das ewige Leben (Haas [2007a]) aus der Reihe der BrennerKrimis, wird der Erzähler erschossen - wie könnte er also auch einen neuen Roman erzählen. Doch auch dieses erzähltechnische Problem löst Wolf Haas in einem Satz: Sein - wohl auf Drängen von Publikum und Verlag - 2009 erschienener neuer Brenner-Krimi Der Brenner und der liebe Gott beginnt mit dem Satz des (eigentlich toten) Erzählers: „Meine Großmutter hat immer zu mir gesagt, wenn du einmal stirbst, muss man das Maul extra erschlagen.“ (Haas [2015], S. 5).

20 Zitiert nach: Hartmann von Aue: Iwein (2001). 
als ein großer König aus der Vergangenheit, dessen Name noch immer bekannt sei, über den mithin noch heute erzählt werde. Die (vage historische) Vergangenheit wird damit in eine unbestimmte Zeit gerückt, ähnlich wie durch die „schöne Formel ,Es war einmal““, die „,[a]lle europäischen Völker kennen und lieben“21. Mit derartig ,unbestimmten Bestimmungen“ wird gleichzeitig das Immerwährende des Erzählten ausgedrückt, denn: „Was einmal war, hat die Tendenz, immer wiederzukommen." 22

Über Vergangenes zu erzählen, bedeutet dabei immer auch Geschichte in Geschichten zu übertragen - oder möglicherweise dort erst entstehen zu lassen. Dass sich Geschichtsschreibung und Literatur (im engeren Sinne) in dieser Hinsicht nicht frappant unterscheiden, sondern dass sich auch die Historiographie literarischer Verfahren bedient, sich ihrer zwangsläufig bedienen muss, ist spätestens seit Hayden White bekannt. ${ }^{23}$

Tatsächlich ist jede historiographische Darstellung, die mehr zu sein beansprucht als eine tabellarische Synopsis singulärer und kontingenter Daten, aus Gründen der argumentativen Plausibilität und Veranschaulichung angewiesen auf Formen der Ästhetisierung, der Fiktionalisierung. Die historiographische Fiktionalisierung in ihren engen funktionalen Grenzen ist ein strukturelles Symptom für die Wissenschaftlichkeit der historisch-philologischen Disziplinen, da der bloße Rückzug in die Registratur der Weltgeschichte [...] das historische Wissen in einer Partikularität und Kontingenz belassen würde, über die jede literarische Fiktion mitsamt ihres zeitgeschichtlichen Informationsgehaltes [sic!] immer schon hinaus ist. ${ }^{24}$

Denn etwas zu erzählen bedeutet, es in Zusammenhänge, in Strukturen zu bringen; diese ,Ordnung ' der Ereignisse dient der Sinn-Schaffung und auch dem Gedächtnis. Ob „den Ereignissen

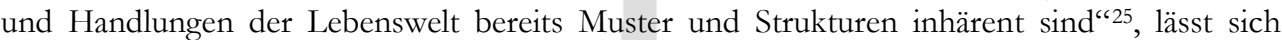
letztlich kaum logisch entscheiden, denn bereits das Formulieren, das ,In-Worte-Fassen“ ist ebenfalls ein „Informationsverarbeitungsprozess“26 wie die Rezeption von Texten, innerhalb dessen „die im Text enthaltenen Informationen mit (schematisch organisiertem) Weltwissen u. a. angereichert werden, so dass in der Rezeption Informationen entstehen, die über die im Text enthaltenen manifesten Bedeutungsinhalte hinausweisen“"27. Damit muss Viktor Laus Aussage noch verschärft werden, denn auch die „Registratur der Weltgeschichte“ in Form einer „,tabellarische[n] Synopsis“ beinhaltet bereits Auswahl und v. a. Ordnungsmuster, die Geschehen in Zusammenhänge bringen und interpretieren. Es gibt somit „,keine Möglichkeit [...], in der Darstellung von ,Realität' der Narrativität zu entkommen“" 28 - selbstredend auch nicht in der Darstellung vergangener ,Realität‘. Der Begriff der historia wird bereits im Mittelalter zum Teil auch

21 Lüthi (1998), S. 35

22 Lüthi (1998), S. 35. Hin und wieder gibt es aber durchaus deutlichere, wenn auch indirekte Anhaltspunkte wie etwa im Wigalois Wirnts von Grafenberg. Der Erzähler bezieht sich hier ausdrücklich auf einen Augenzeugen, der ihm die Geschichte berichtet habe (W 595-597; 11686-11690; vgl. Wirnt von Grafenberg [2005]); das bedeutet, die Handlung - und damit die Lebenszeit des Königs Artus - kann maximal ein paar Jahrzehnte zurückliegen. Zur variablen Relation zwischen Geschichtsschreibung und Augenzeugenschaft im Laufe der Geschichte vgl. Luraghi (2014).

23 Vgl. z. B. White (1986), White (1990). Zum Verhältnis von „Geschichtsschreibung und Dichtung“ aus Sicht der (älteren) Literaturwissenschaft vgl. beispielsweise Ebenbauer (1985; Zitat S. 53).

24 Lau (1999), S. 520f.

25 Brüggemann (2008), S. 369, mit Bezug auf Ricœur (1988, 1989, 1991).

26 Brüggemann (2008), S. 368.

27 Ebd.

28 Wolf (2002), S. 8. Wolf weist auch explizit darauf hin, dass „der Prozeß der Literarisierung von Geschichte von den Erlebnisstrukturen des Interpreten abhängt, die Vergangenheit immer wieder in neuen Konfigurationen geordnet wird und deswegen Geschichte - nicht nur wegen aktueller politischer Erfordernisse - immer wieder umgeschrieben werden muß“ (ebd., S. 4). 
so umschrieben, dass in ihr „mittels eines heuristischen Verfahrens die Vergangenheit geordnet und narrativ aufbereitet wird" 29 , Ordnung und Narration also integrale Bestandteile sind. Entsprechend konstatiert Hans-Werner Goetz:

In einem gewissen Sinn war folglich bereits dem mittelalterlichen Verständnis von Geschichte bewußt, daß erst die Darstellung „Geschichte“ macht (eine Erkenntnis, die uns erst seit wenigen Jahrzehnten wieder ins Bewußtsein gerückt ist), ohne daß freilich dieser Spiegelungscharakter, die Vergangenheit mit den Augen der Gegenwart zu sehen, erkannt oder reflektiert worden wäre. ${ }^{30}$

Die - historiographischen wie literarischen - Texte überliefern freilich nicht Geschichte (auch mündliche Erzählungen über Vergangenheit nicht), sondern, Geschichts-Bilder ${ }^{6}$, d. h. die jeweils „persönlich-subjektiv gefärbte Sicht von der Geschichte“31, genauer von „Segmente[n] der Geschichte“32. Im Gegensatz zum ,Geschichtsbewusstsein', das nach Hans-Werner Goetz ,jeder historischen Erzählung [zugrunde] liegt“"33, konstituiert sich das Geschichtsbild, das wir in den Texten greifen können, erst durch das Erzählen:

Wohl aber ist die Erzählung mit Rüsen eine entscheidende Ausdrucksform des Geschichtsbewußtseins, das „die erinnernd vergegenwärtigten zeitlichen Veränderungen der Vergangenheit als kontinuierliche Verläufe darstellt, an die die Zeiterfahrung der Gegenwart deutend angeschlossen und in eine Zukunftsperspektive hinein extemporiert werden kann“.34

Dass Texte, speziell literarische Texte, auch dazu dienen, Ereignisse in Erinnerung zu behalten, bzw. dass zentrale Geschehnisse nicht zuletzt in literarische Form gebracht werden, um weitererzählt werden zu können, gehört wohl zu den anthropologischen Grundgegebenheiten. Gewisse Ur-Erfahrungen beispielsweise scheinen dabei weltweit verbreitet zu sein: So gibt es etwa bei zahlreichen Völkern Erzählungen über eine große Flut, eine Sintflut, von Gott oder den Göttern geschickt, deren „Wucht [...] über die Menschen hinweg [geht]“35 und alle Menschen vernichtet bis auf einen Auserwählten wie Noah oder Uta-napischti aus dem Gilgamesch-Epos, der als neuer Stammvater das menschliche Leben auf Erden fortsetzen darf. ${ }^{36}$ Die Erinnerung an Vergangenes ist dabei von vorneherein problembehaftet, wenn man ein ,historisch richtiges Bild der Ereignisse erwartet. Wie Johannes Fried vor dem Hintergrund der Neurowissenschaften gezeigt hat, bedeutet Erinnern von Beginn an immer auch „Selektion und Vergessen““37, sodass die Quellen der Geschichtswissenschaft wie Erinnerungen von Personen, aber auch historiographische Zeugnisse den Erinnerungsprozess schon allein durch die (zunächst unbewusste) Auswahl des Erinnerten steuern und auch der Akt des Erinnerns jeweils ein ,an das gegenwärtige Leben selbst gebundener und von seinen momentanen Bedürfnissen gelenkter Prozess ist“" 38 ; dies steht im Zusammenhang mit dem „konstruktive[n] Aspekt von Erinnerung: Sie hilft, die Ge-

29 Wolf (2016), S. 14; vgl. zur Begriffs- bzw. Gattungsdefinition von ,Chronik‘ ebd., S. 12-31. Vgl. auch die begriffsgeschichtliche Darstellung zu ,geschiht' und ,historia ‘ bei Knape (1988).

30 Goetz (2008), S. 99.

31 Goetz (2008), S. 16.

32 Goetz (2008), S. 15.

33 Goetz (2008), S. 21.

34 Goetz (2008), S. 21f., mit Bezug auf Rüsen (1983), S. 55.

35 Das Gilgamesch-Epos (2012), S. 144.

36 Vgl. Genesis 6,1-9,29 und den dazu gehörenden Kommentar (Die Bibel [1998], S. 22). Zum Mythos der Sintflut und zur These der Überflutung des Schwarzen Meeres durch Wassermassen aus dem Mittelmeer vgl. Haarmann (2003), bes. S. 20-30, und den Sammelband von Mulsow/Assmann (2006).

37 J. Fried (2004), S. 15.

38 J. Fried (2008), S. 7 (Herv. i. O.). 
genwart wahrzunehmen, gibt ihr Sinn und ordnet sie zwischen Vergangenheit und Zukunft ein; als solche produziert sie Identität und Kontinuität; ja, nur durch sie kann die Wirklichkeit Gestalt annehmen“"39. Dieser ,Konstruktionsprozess' geht so weit, dass das (kulturelle) Gedächtnis ,Erinnerungen“ an Ereignisse enthält, die nie stattgefunden haben, dass aus „Pseudoerinnerungen“ „Gedächtnisimplantate“ ${ }^{“ 40}$ werden. Hinzu kommen - sowohl bei historiographischen als auch bei literarischen Texten - bewusste Einflussnahmen auf das Dargestellte, sei es beispielsweise aufgrund von politischen Zielen oder durch Literarisierungsprozesse, in denen ,Erinnerung literarisch umge- oder überformt wird.

Das Verhältnis von Literatur und Gedächtnis ist ein vielfältiges. ${ }^{41}$ Astrid Erll und Ansgar Nünning unterscheiden drei „Grundrichtungen“44:

a) das „Gedächtnis der Literatur“: Literatur bringt durch systemimmanente Bezüge inhaltlicher oder auch formaler Art sich selbst immer wieder ins Gedächtnis, d. h. es „werden

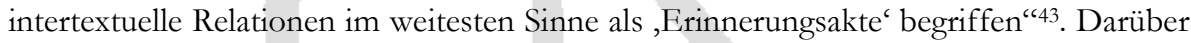
hinaus wird an Literatur gedacht, z. B. in Form von Kanonbildung oder Literaturgeschichtsschreibung. Das bedeutet, Literatur steht zum einen als Symbolsystem, zum anderen als Sozialsystem im Fokus. ${ }^{44}$

b) „Gedächtnis in der Literatur": Das Phänomen des Gedächtnisses wird in zahlreichen literarischen Texten beschrieben und verarbeitet; „Leitfrage [einer Analyse] ist [...], mit welchen Verfahren die Inhalte und Funktionsweisen des Gedächtnisses thematisiert und inszeniert werden ${ }^{\text {“45. }}$.

c) „Literatur als Medium des Gedächtnisses“: Literatur dient als Vermittler von, Gedächtnis“, d. h. „von kulturspezifischen Schemata über Vergangenheitsversionen bis hin zu Vorstellungen von den Funktionsweisen des Gedächtnisses “46. Literatur bietet hierbei auch einen Freiraum etwa für ,alternative Vergangenheitsversionen“47 o. ä.

Für das Verhältnis von Historie und Literatur mit Blick auf Mittelalter und Frühe Neuzeit sind vor allem der erste und der dritte Punkt zentral: Literatur ist ein selbstreflexives Medium, das sich sowohl über Inhalte (intra- und intertextuelle Verknüpfungen) als auch über die formale Seite (Erzählschemata, Gattungstraditionen) immer wieder auf sich selbst bezieht; auf diese Weise wird freilich auch verarbeitete Geschichte in der literarischen Tradition weitergegeben; gerade mittelalterliche und frühneuzeitliche Texte der Geschichtsschreibung (aber nicht nur diese) sind in mehrfacher Hinsicht „Hybride“48. Daneben bewahrt Literatur Vergangenes auf,

39 François/Schulze (2009), S. 14

40 J. Fried (2008), S. 2; vgl. dazu auch J. Fried (2003) und umfassend J. Fried (2004). Ein solches Gedächtnisimplantat wird im Zusammenhang mit Friedrich I. Barbarossa und dem sog. Frieden von Venedig in Kap. 3.5 beleuchtet.

41 Es geht dabei im Folgenden nicht in erster Linie um das ,kollektive Gedächtnis', da sich darüber aus einzelnen Texten kaum Aussagen gewinnen lassen. Einen kritischen Überblick über die „Theorien des kollektiven Gedächtnisses“ bietet Robbe (2009), S. 41-79.

42 Erll/Nünning (2005), S. 3.

43 Erll/Nünning (2005), S. 3.

$44 \mathrm{Zu}$ verschiedenen Strategien und dem Legitimationscharakter des Erinnerns an Literatur in Literatur am Beispiel des Tristan Gottfrieds von Straßburg vgl. Kellner (1999b).

45 Erll/Nünning (2005), S. 4.

46 Erll/Nünning (2005), S. 5.

47 Erll/Nünning (2005), S. 7.

48 Wolf (2016), S. 28. Wolf verwendet den Begriff hier in Bezug auf Werke der Gesta-Tradition, in denen „Teile einer Welt- oder Reichschronik enthalten sein“ können, doch die Vermischung betrifft zum einen nicht nur Geschichten von Stämmen oder Völkern und zum anderen nicht nur weitere historiographische ,Gattungen‘; „der Übergang zwischen Chronik und Epos“ (ebd., S. 2) ist bereits in der Geschichtsschreibung in Mesopotamien fließend und 
wandelt es aber auch - evtl. in Rückgriff auf Erzählschemata o. ä. - ab oder sogar in Neues um. Überdies ist das ,Gedächtnis in der Literatur' von Relevanz, freilich weniger im Sinne einer literarischen Darstellung bzw. Diskussion von biologischen oder psychologischen Funktionen von ,Gedächtnis‘, ${ }^{49}$ jedoch in Hinblick auf die - oft explizite - Funktionalisierung von Literatur für das Gedenken, die gedechtnus, die sich auch intradiegetisch spiegeln kann. ${ }^{50}$

Im Sinne der gedechtnus erfüllen (literarische wie chronikalische) Texte durch die in ihnen stattfindende Aktualisierung der Geschichte eine wichtige Transfer-Funktion von der Vergangenheit in die (jeweilige Rezeptions-)Gegenwart und letztlich als schriftlich fixierte Texte in deren Zukunft. So blieb nicht nur Friedrich I. Barbarossa bis heute im Gedächtnis, sondern auch der Sänger Tannhäuser oder Theoderich der Große sind als Sagenfiguren noch greifbar, wenngleich die historischen Gestalten freilich wohl (fast) nur Fachkreisen bekannt sind. Doch diese - die Fachkreise - gab und gibt es parallel zu den überformten Erzählungen, sodass beide Formen von ,Geschichte $/ \mathrm{n}^{\star}$ auch gegebenenfalls in ihrer Widersprüchlichkeit nebeneinander existieren. Dabei kann das Wissen der Historiker bzw. Historiographen durch Sagentraditionen ergänzt werden - das Wissen um Leben und Taten Friedrichs I. Barbarossa, wie es etwa jüngst Knut Görich umfassend dargestellt hat, ${ }^{51}$ widerspricht letztlich nicht zwangsläufig einer Vorstellung von der Wiederkehr des Kaisers (wenn man an eine solche überhaupt glauben möchte) - oder aber es können auch durchaus Unstimmigkeiten bzw. sogar Unvereinbarkeiten bemerkt und konstatiert werden: Frutolf von Michelsberg „,verweist als erster auf chronologische Widersprüche zwischen der Darstellung des Jordanes und sagenhaften Überlieferungen“ in Bezug auf Theoderich den Großen, Ermanarich und Attila, die einerseits „als Zeitgenossen dargestellt“52 würden, andererseits (wie etwa bei Jordanes) nicht. ${ }^{53}$ Zentral ist hier wie in anderen Fällen auch,

nicht nur im Mittelalter „kann man die [...] historischen ,Gattungen“ nicht sinnvoll nach Geschichtsschreibung und -dichtung trennen“ (ebd., S. 22).

49 Die Diskussion um die Tätigkeit des Gedächtnisses als „Konstruktionsarbeit“ (Schmidt [1991], S. 378; im Gegensatz zur Aufbewahrung von Erinnerungen) vor dem Hintergrund kognitionswissenschaftlicher Ansätze stellt knapp Schmidt (1991) dar.

50 Tilmann Robbe unterscheidet - aus der Perspektive der Gegenwart nicht ganz zu Unrecht, aber sprachlich unscharf - methodisch deutlich zwischen ,Erinnerung' und ,Gedächtnis'; während ,Erinnerung` eine Tätigkeit sei, damit der aktive Part, stelle Gedächtnis den passiven Speicher dar. „Dem entspricht, dass der Erinnerung das verbale ,erinnern' zur Seite steht, während dem Gedächtnis die Verbform fehlt: Dem Gedächtnis entspricht keine Tätigkeit, und das Gedenken mit seinem korrespondierenden Verb setzt einen anderen Schwerpunkt." (Robbe [2009], S. 32). Abgesehen davon, dass es sich sowohl bei ,Erinnerung' als auch bei, Gedächtnis` um von Verben abgeleitete Abstrakta handelt, kann den neuhochdeutschen Wörtern zwar durchaus semantische Differenz zugestanden werden, die so aber nicht auf das frühneuhochdeutsche Wort gedechtnus zutrifft, das etwa ,erinnerndes Gedenken' bedeutet und nicht mit dem modernen Konzept eines Gedächtnisses gleichzusetzen ist. Die Spannbreite des Begriffs macht JanDirk Müller anhand der Verwendung in den von Maximilian I. in Auftrag gegebenen Werken deutlich: gedechtnus bedeutet das Bewahren von Traditionen, im feudaladeligen Kontext v. a. ,dynastisch-genealogische[r] Traditionen“, richtet sich also auf das Herkommen und dessen Weitertradierung. Entsprechend „meint [gedechtnus] historische Forschungen, literarische Fassung der Memoiren und beider bildliche Ausstattung“" (J.-D. Müller [1982], S. 80). Gedecbtnus hat damit immer auch eine Legitimations- und Stabilisierungsfunktion (vgl. J.-D. Müller [1982], S. 81).

51 Vgl. Görich (2011).

52 Lienert (2010), S. 36. Vgl. zur Äußerung Frutolfs von Michelsberg Ekkehardi Chronicon Universale (1844), S. 130; die Chronik Frutolfs galt als verloren, bis 1896 nachgewiesen wurde, „daß diese Chronik in dem bis 1098 reichenden, wenn auch z. T. getilgten Teil einer mit 1106 endenden Chronik, die bis dahin als Ganzes als die Ekkehards von Aura galt, im Autograph vorliegt“" (Schmale [1979], S. 995). Vgl. zu Jordanes Schwarcz (2002) sowie zum Verhältnis von Dietrich-Sage und Theoderich-Historiographie mit besonderem Blick auf die Weltchronik Heinrichs von München Kornrumpf (1985).

53 Die Relation von historiographischen und im engeren Sinne literarischen Zeugnissen durch elf Jahrhunderte kann anhand der Zusammenstellung der, Testimonien` zu Dietrich von Bern/Theoderich dem Großen durch Elisabeth 
dass im Mittelalter „die Sage durchaus als potentielles Zeugnis ernst genommen“54 wird, denn „[s]elbst die Sagenkritik lässt darauf schließen, dass die Verbindlichkeit von Sage nicht einfach ignoriert werden konnte“ 55 .

Gedenken und Erinnerung hinwiederum sind zentral für Identitätsbildung nicht nur des Einzelnen, sondern auch von Gruppen oder Nationen; ${ }^{56}$ Traditionsbildungen über ein (gemeinsames) „Bild der Vergangenheit“ „,sind für jede Gemeinschaftsbildung wohl unabdingbar“57. Wie groß die Rolle der Literatur dabei ist bzw. sein kann, zeigt etwa die Suche nach dem ,deutschen Wesen' im 19. Jahrhundert, die nicht zuletzt eine Suche nach ,deutscher Literatur' war. Die Berufung auf eine gemeinsame kulturelle Vergangenheit und hierbei insbesondere auf literarische Werke wie das Nibelungenlied oder Wolframs von Eschenbach Parzival war für die Entstehung eines deutschen Nationalgefühls zentral. ${ }^{58}$ Auch die (sagenhafte) Figur des wiederkehrenden Kaisers Barbarossa spielte in dieser Zeit eine große Rolle, ${ }^{59}$ wurde „,von den Deutschen [...] als imperialer Gipfelpunkt imaginiert ${ }^{\text {"60 }}$ und erlebte tatsächlich eine literarische Wiederkehr in den Jahren der Wende bzw, der deutschen Wiedervereinigung, wenn auch in völlig anderer Form. ${ }^{61}$ Dies bedeutet auch, dass ,,alle Formen der gedechtnus" (bei Wolf speziell die Herkunftssage) ,auf die Gegenwart ziel[en], ja daß sogar im Wege eines ,Präsentismus“ die Anforderungen der Gegenwart bei der Darstellung der Geschichte immer mit einbezogen werden“62.

Literatur ist demnach Träger sowohl von Gedächtnis- bzw. Erinnerungskonzepten als auch von ,Techniken', Erinnerung hervorzurufen, sowie von Erinnernswertem. Die Analyse der genannten Aspekte in literarischen Werken einer vergangenen Epoche kann damit Aufschluss geben über Vorstellungen von Gedächtnis, über Wege der Verknüpfung mit Vergangenem und über den Umgang mit der Vergangenheit selbst. Der Akt des Erzählens selbst bzw. die „Erzäblung stellt ein grundlegendes kulturelles Phänomen dar"633, das als Bindeglied zwischen Erinnerndem und dem Erinnerten, zwischen Gegenwart und Vergangenheit also, das Bild eben dieser Vergangenheit (und damit auch das der jeweiligen Gegenwart) abbildet und zum Teil zugleich prägt. Literatur dient so auch der gedechtnus, der „Sicherung von Tradition“ “64, indem die Erinnerung an Ereignisse, Handlungen oder Personen durch sie wach gehalten wird. Das Vergangene, also die Geschichte, ,, ist, wie es in einer oft zitierten Äußerung Ciceros heißt, das ,Leben des Gedächtnisses“ (bistoria vita memoriae) "65 und darüber hinaus - ebenfalls nach Cicero - magistra

Lienert nachvollzogen werden, wobei dort aus pragmatischen Gründen historiographische Quellen „,nur in repräsentativer Auswahl aufgeführt" sind (vgl. Lienert [2008], Zitat S. 8).

54 Lienert (2010), S. 36

55 Lienert (2008), S. 12

56 Vgl. dazu Neumann (2005).

57 Graus (1975), S. 2 (Herv. i. O.). Graus benennt die Bedingungen, die erfüllt sein müssen, um von solchen, Traditionen' sprechen zu können: 1. Projektion in die Vergangenheit bei gleichzeitiger Relevanz für die Gegenwart; 2. räumliche und zeitliche Fixierung; 3. Bestand der ,Tradition` über mehrere Generationen; 4. keine Begrenzung auf „rein gelehrte Forschung“ (S. 7; vgl. S. 6f.).

58 Vgl. zur Rezeption des Nibelungenliedes im 19. Jahrhundert Ehrismann (1975) und Heinzle/Waldschmidt (1991).

59 Vgl. Kaul (2007).

60 Schneidmüller (2007), S. 75.

61 Vgl. dazu Schindler (2017).

62 Wolf (2002), S. 37.

63 Scharfenberg (2011), S. 13 (Herv. i. O.).

64 J.-D. Müller (1982), S. 80.

65 Burke (1991), S. 289, mit Bezug auf Cicero (vgl. Cicero [1965], S. 245: „Historia vero testis temporum, lux veritatis, vita memoriae, magistra vitae, nuntia vetustatis, qua voce alia nisi oratoris immortalitati commendatur?"). 
vitae. ${ }^{66}$ Historiographie und andere Formen des Erzählens von Geschichte funktionalisieren das vergangene Geschehen damit im Konnex von Vergangenheit und Gegenwart etwa „als leuchtendes Beispiel oder aber als Warnung“"67. Und „[d]er Mensch ist selbst Glied dieser Geschichte, die bis an die Gegenwart heranreicht und in die Zukunft hinein weiterwirken wird, so daß er mitten in einem Prozeß steht, der die Vergangenheit für die Gegenwart aufbereitet und diese selbst wieder ,Geschichte` werden läßt“"68. Literatur als Medium eines solchen, Weiterwirkens lässt damit Tradition und Geschichte entstehen.

Aus diesem Dreischritt - Vergangenheit, Gegenwart, Zukunft - ergeben sich drei zu beachtende Implikationen des Erzählens von Geschichte. Zum Ersten steht hinter dem jeweiligen Werk das Geschichtsbewusstsein des Autors, das (in Teilen) daraus zu erschließen ist; ein umfassenderes Bild ist letztlich nur dann zu erhalten, wenn mehrere Texte eines Autors überliefert sind. Zum Zweiten schreibt der Verfasser für ein intendiertes Publikum (beispielsweise Fachleute oder Laien) und möglicherweise für einen Auftraggeber; dadurch stehen unterschiedliche Intentionen hinter den Texten, denn: „Interesseloses Umgehen mit historischen Ereignissen und Persönlichkeiten gibt es nicht." ${ }^{“ 69}$ Geschichte wird nicht um ihrer selbst willen erzählt, sondern jeweils für eigene Zwecke eingesetzt. Zum Dritten spielt bei jeder Lektüre das Geschichtsbewusstsein des jeweiligen Rezipienten eine Rolle. ${ }^{70}$ Bei der Analyse eines Textes über Geschichte oder Geschichtliches muss demnach zunächst - sofern möglich - dessen Hintergrund (Auftraggeber, Verfasser, intendiertes Publikum) geklärt ${ }^{71}$ und das eigene Geschichtsbild, das jeder (moderne) Rezipient mitbringt, möglichst bewusst gemacht und in den Hintergrund gerückt werden.

Autor, intendiertes und tatsächliches Publikum haben jeweils Vorstellungen von der,Geschichte` und damit vom, Wahrheitsgehalt` des Erzählten, vom Verhältnis von ,Fakt` und ,Fiktion` und die Erzählung selbst transportiert durch ihren Konstruktionscharakter, den jedes Erzählen bereits durch eine Ordnung des Erzählten impliziert, über den Text hinausgehende Bedeutung(en) und auch Sinnzuschreibungen. Daraus folgt, dass sich allein durch den Akt des Erzählens das Erzählte bereits vom Geschehenen entfernt. Durch Intention oder (im Falle der Heldenepik beispielsweise Jahrhunderte lange) Erzähltradition kann die Distanz zwischen ,Fakt und ,Fiktion' letztlich so groß werden, dass das historische Geschehen nicht mehr als solches im (literarisch) Erzählten erkannt werden kann.

In diesem Spannungsfeld ist die Sage angesiedelt, die zwar - wie das Märchen - häufig „mag[ische], numinose[] oder myth[ische] Elemente[]“72 besitzt, aber dennoch einen Anspruch auf Glaubwürdigkeit erhebt, der sich nicht zuletzt aus einer genauen lokalen und/oder zeitlichen Verortung und auch aus Verweisen auf ,heute' noch sichtbare Artefakte speist, durch die eine

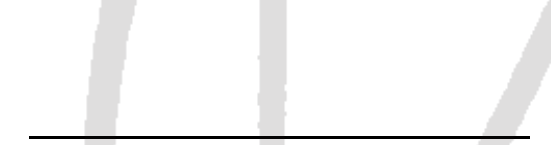

66 Vgl. Anm. 65. František Graus stellt heraus, dass gerade diese Formel im Mittelalter zwar bekannt war, aber vor allem in der Frühen Neuzeit bzw. im Humanismus „,recht populär“ wurde (vgl. Graus [1987], S. 18).

67 Graus (1987), S. 11. Graus benennt darüber hinaus neben der Belehrung durch Vergangenes und Bewahrung von Vergangenem auch noch die Legitimationsfunktion, die Geschichtsschreibung etwa im Kontext von Rechtsansprüchen haben kann (vgl. Graus [1987], S. 23).

68 Goetz (2008), S. 14.

69 Brunner (1997), S. 31.

70 Vgl. Goetz (2008), S. 26.

71 Ein Problem, das sich bei anonym überlieferten Texten durch gänzlich fehlende Daten zum Autor noch potenziert, liegt darin, dass ,,[h]äufig [...] der Anteil von Verfassern, Übersetzern, Bearbeitern und Druckern nicht auseinanderzuhalten [ist]" (Braun [2004], S. 321).

72 Schweikle (1990), S. 405. 
Verbindung zur jeweiligen Rezeptions-Gegenwart hergestellt wird. ${ }^{73}$ Sie steht gewissermaßen am einen Ende der Möglichkeiten, Geschichte zu erzählen, während das andere Ende (theoretisch) die rein faktische Historiographie bildet. Jeder Autor von Historiographie oder von, Geschichten über Geschichte' muss sich - bewusst oder unbewusst - auf dieser Skala positionieren, wobei er sich mit seinem Werk in einer mehr oder weniger großen Distanz zum Geschehen befinden kann und damit selbst bereits (u. U. zwangsläufig) auf eine Rezeptionskette zurückgreift. Wer etwa heute über Barbarossa schreibt, kann kaum umhin, die Kyffhäuser-Sage zumindest zu erwähnen - denn nicht zuletzt durch sie ist Barbarossa zu einer der bis heute bekanntesten historischen Persönlichkeiten des europäischen Mittelalters geworden und so speist sich seine Bedeutung auch daraus.

Die Berufung auf eine oder mehrere Quellen ist schon in der hochmittelalterlichen Literatur topisch geworden und dient der Legitimierung und v. a. der Versicherung der veritas. ${ }^{74} \mathrm{Im}$ Kontext von historiographischem Schreiben kommt den Quellen natürlich ein anderer Stellenwert zu als bei ,fiktiven' Werken (wenn sich etwa Gottfried von Straßburg auf die einzig ,richtige Tristan-Version, die des Thomas von Britannie, beruft ${ }^{75}$, wenngleich in beiden Fällen nicht nur ,Wahrheit' für das Gesagte resp. Geschriebene in Anspruch genommen, sondern zudem auch dessen Nachprüfbarkeit behauptet wird. Darüber hinaus spielt in diesem Zusammenhang sicherlich auch die sprachliche Form - Prosa vs. Vers - eine Rolle, denn „[d]ie Verwendung von Prosa für nichtfiktionale Textsorten besitzt zu diesem Zeitpunkt [der Entstehung der ersten Prosaromane; A. S.] bereits eine lange Tradition“"76. Rüdiger Schnell hat zwar die These, für Spätmittelalter und Früher Neuzeit gelte: Prosa transportiere ,Wahrheit', Vers dagegen sei das „Medium der Lüge ${ }^{\text {“77 }}$, bereits 1984 deutlich relativiert, doch im Gegensatz zu Prosatexten, bei denen diese Tendenz zugunsten der Schriftlichkeit deutlich zurückgeht, changiert ein Vers-Roman wie Wolframs Parzival dennoch zwischen Verweisen auf Gehörtes und Gelesenes, zwischen Mündlichkeit (u. a. des Vortrages) und Schriftlichkeit. ${ }^{78}$ In der Zeit des Buchdrucks, der eine breitere Wirksamkeit von Schriftlichkeit ermöglicht, steigt auch für literarische Werke deren Stellenwert, selbst wenn zum einen die stille Lektüre noch lange nicht, wie heute, die mehr oder weniger einzige, ,normale Rezeptionsvariante darstellt und in literarischen wie historiographischen

Solche Strategien zur Legitimation des Erzählten sind freilich nicht auf die Sage beschränkt, sondern - naheliegenderweise - etwa auch in historiographischen Kontexten verbreitete, aber ebenso in fiktiv(er)en Erzählungen wie etwa im Eulenspiegel, wenn der Erzähler nicht nur detailliert die verschiedenen Orte benennt, an denen der Protagonist sein Unwesen getrieben hat, sondern gelegentlich auch darüber hinausgehende Informationen zum gegenwärtigen Stand der Dinge gibt, die für die Handlung selbst irrelevant sind: „Und Dil von Uetzen, der Burger zu Ampleven, ward sein [Ulenspiegels; A. S.] Tauffpfetter. (Und Ampleven ist daz Schloß, daz die von Magdburg etwan vor funnfftzig Jaren mit Hilff der andern Stät für ein böß Raubschloß zerbrachen. Die Kirchen und daz Dorff dabei hatt nun der wirdig Arnolff Pfaffmeier, Apt zu Sunten Ägidien.) “(Ein kurtzweilig Lesen von Ulenspiegel [2001], S. 10).

74 Zur Tradition der Quellenberufung und ihrer Entwicklung im Mittelalter vgl. Grubmüller (1995), S. 43ff., Haug (2003a) und Haug (2003b); eine umfangreiche Sammlung von Quellenberufungen bietet Lofmark (1981).

75 Vgl. Gottfried von Straßburg: Tristan (2004), V. 131-154.

76 Bennewitz (1991), S. 151.

77 R. Schnell (1984), S. 215.

78 Vgl. dazu Groos (1995). Auf die Rolle der Schrift bei der Vermittlung von Tradition/Geschichte durch die durch Verschriftlichung ermöglichte Distanzierung und Historisierung hat J.-D. Müller hingewiesen (vgl. J.-D. Müller [1982], S. 83). 